\title{
METHODS FOR AUTOMATIC IDENTIFICATION OF COKE OVENS FOR AUTO POSITIONING SYSTEMS IN COKE PLANT OF STEEL INDUSTRIES
}

\author{
Prabal PATRA ${ }^{1}$, Chitresh KUNDU ${ }^{2}$, Arunangshu GHOSH ${ }^{3}$, Bipan TUDU ${ }^{4}$, Rajib BANDYOPADHYAY \\ ${ }^{1}$ TATA Steel, Instrumentation \& Control, prabal.patra@ tatasteel.com \\ ${ }^{2}$ TATA Steel Instrumentation \& Control, chitresh.kundu@tatasteel.com \\ ${ }^{3}$ NIT Patna, arunangshu.ghosh@gmail.com \\ ${ }^{4}$ Jadavpur University, bipantudu@ gmail.com \\ ${ }^{5}$ Jadavpur University, rb@iee.jusl.ac.in
}

\begin{abstract}
Blast furnace operation requires highest quality raw materials, operation and operators. Among them coke is the most important, in terms of its effect on operation and hot metal quality. A good grade coke produces highest thermal energy and it is highly efficient in case of metal reduction. Usage of good grade coke to blast furnace will ensure lower coke rate, higher productivity and lower hot metal cost. In order to concentrate the carbon in coke the coke making process involves carbonation of coal to high temperature $\left(1100^{\circ} \mathrm{C}\right)$ in oxygen free atmosphere. There is need to automate the coke oven operations as much as possible. In order to improve the level of control and management of coke oven, the research on intelligent control system is carried out. In modern advanced control system of coke oven, the control scheme of combination, feedback \& feed-forward merged with management are widely utilized. The integrated management and control system of coke oven is introduced systematically, including the production plan and scheduling management (Dynamic scheduling) $\&$ heating control system (Mathematical modeling) i.e. evaluating battery temperature, intelligent combustion control and pressure control of gas collector of coke oven battery.
\end{abstract}

Keywords: LASER, image processing, RADAR, RFID, coke ovens

\section{INTRODUCTION}

Coking is a process of carbonising coal into coke in high temperature in the coke oven. When coke is matured / prepared, coke cake will have some constriction and the centre temperature will reach up to $900 \sim 1100$ degree centigrade. If the centre temperature cannot reach the required temperature, the coke cake will be incompletely cooked and does not prepare well. This will affect coke oven battery service life seriously. If the centre temperature higher than the required range, the coke cake is easy to brake. This will affect the quality of material (coke). There are three stages in the whole operation as e.g. running (heating), pushing and setting off. This means that coke pusher, runs to the appointed (ready to push) oven to push according to the predefined programming. Therefore, pushing coke should be operated according to definite sequence and the designing model of this sequence transforms to push/charge plan model. In a large coke oven most operators placing coke pushers are in the right position not just by vision and experience but with the help of some identification and positioning system. Sometimes the coke pushers are not aligned properly at ready oven centre. Hence the master controller/PLC/DCS must operate continuously, the long travel drive must be restarted and stopped continuously, and the hydraulic brake must be restarted continuously to place the machine in a proper position. The coke pushing/charging plan is still adopted manually and the work is trivial and stiff. Therefore, it is very essential to arrange the pushing/charging plan automatically and give the coke pushing/charging plan timely.

To run the coke making process safely and efficiently few conditions are imposed on the movement of these machines. Identification of the coke oven helps in the auto positioning of the door extraction cars which in turn extract the door of the oven to push the converted coke into the bin. To achieve this condition precise oven identification is essential for each machine. Similarly machine interlocking would be mandatory to operate these machines. These interlock logics are implemented in the machine PLCs for preventing any untoward incident like wrong pushing.

Coke plants are one of the highly challenging areas in steel plants and to automate the identification of the coke ovens, different techniques are used. The major limitation of coke plant automation is the extreme environmental conditions prevalent in the plant location such as high temperature, humidity and so on. Identification of oven position from each machine is very difficult considering the harsh environment in which these machines operate. The solution should be rugged 
enough to withstand the rough environment like heat, fumes, dust, jerk and vibration. Several solutions have been developed over the time to achieve oven identification. In this paper we have identified and proposed different solutions developed for oven identification and automatic positioning of coke-oven machines.

The paper is organised as follows. At first, the problem is explained. In section 1 Marker plate reader/Sensor is discussed. In section 2, RF based identification is explained. Imaging based oven identification is discussed in section 3 . Section 4 is about a Radar based approach towards the problem. In section 5 a comparative study of the four approaches is given.

\section{PROBLEM DEFINITION}

In Coke plant automation, it is essential to know the position of the moving machines with respect to the oven position. This can be achieved by applying different approaches such as sensor driven, RFID based, imaging, RADAR based approaches etc. This paper introduces various approaches towards oven position identification and a comparative study on such systems.

\subsection{Marker plate reader/Sensor [AUTOSPOT] based Oven Identification}

In Coke plant automation, it is essential to know the position of the moving machines with respect to the oven position. Hence, an automatic positioning system (AUTOSPOT) has been developed for enabling rail borne vehicles, such as coke plant machines, to be driven accurately to the defined positions. This system identifies the predefined position such as oven number and its alignment status with respect to the defined position. The system consists of four important components.

Sensing unit (reader): This is fixed with moving machine and consists of eight Infrared transmitters and receivers mounted opposite to each other and two for edge detection. The Autospot sensor unit has been shown in the Fig. 1.

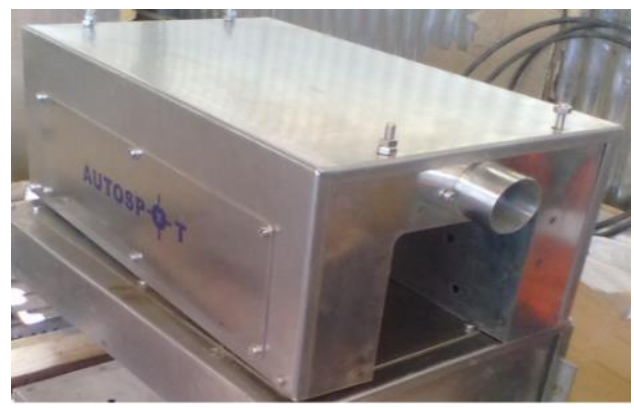

Fig. 1. Autospot Sensor unit
Marker plates: This is a stainless steel rectangular plate consists of binary coded rectangular holes along the length of the plate. These holes represent the oven identification number and are read using the sending unit (reader). The marker plate is designed to take into account the sway of machine and the variation in elevation. The transmitter heads are mounted on one side of the $U$ - tunnel that runs through the sensing element and the receiver heads on the other. The transmitter sends a modulated IR beam which after passing through the indexed plate is received by the array of receiver. In addition to the serial data the information about the left and right alignment is also sent through a potential free contact. A Typical Binary Coded marker plate has been shown in the Fig. 2.

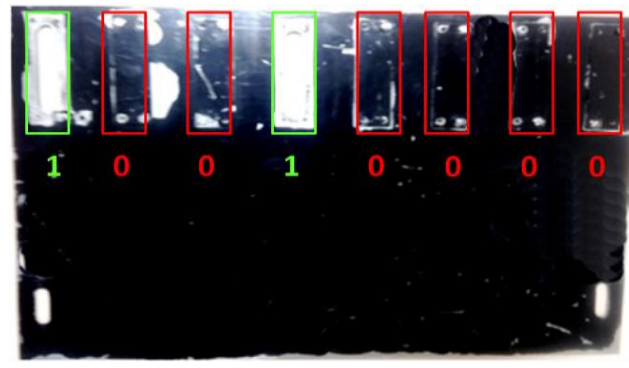

Fig. 2. Binary Coded Marker plate

Dummy encoder wheel: Apart from Auto spot, incremental encoders are used to park the machines with fine accuracy. These encoders are installed on a measuring wheel, a dummy wheel (Shown at Fig. 3) which moves with the machine.

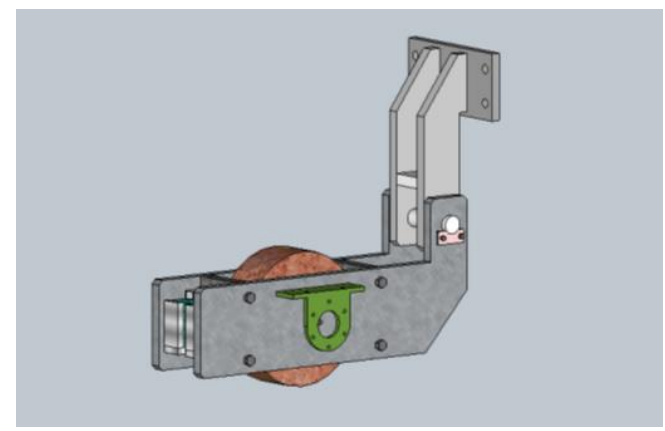

Fig. 3. Measuring wheel

Embedded Communication Card: It is an embedded platform (AUTOSPOT communication module as shown in Fig. 5) which reads the binary number from the marker plate from the infrared sensors. The arrangement of the IR sensors in the sensing unit has been shown in the Fig. 4.

As the sensing unit moves over the marker plates, the status of the infrared sensor corresponds to the binary value of oven identification number. This information is read by the microcontroller in the AUTOSPOT communication module (as shown in Fig. 5) and passed on to the PLC through RS485 link through a telegram as shown in Tab. 1. 
AutoSPOT Sensor Arrangement:

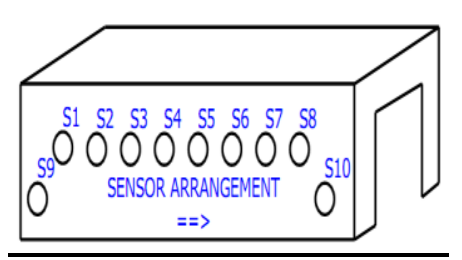

1. Sensors $S 1$ to $S 10$ are connected to inputs 11 to 110 of AutoSPOT.

2. Direction Sensors ( $\$ 9$ and $S 10$ ) represents left or right movement.

3. Sensors $S 1$ to $S 8$ represents 8-bit unique OvenID.

Left Right

Fig. 4. Autospot sensor arrangement schematic

Tab. 1. Autospot telegram format AutoSPOT Telegram Format

\begin{tabular}{|c|c|c|c|c|c|c|c|c|c|c|}
\hline 1 & 2 & 3 & 4 & 56 & 7 & 8 & $g$ & 10 & 11 & 12 \\
\hline HDR & TGIDinio & Marval & PtAdr & Free Fre & CROHigh & CRCLOW & Untild & FTR1 & FTR2 & FTR3 \\
\hline OXAA & ** & * & * & $0 \times 550 \times 0$ & \#* & *t & $*$ & $0 \times 20$ & $0 \times 00$ & OXOA \\
\hline
\end{tabular}

The header/footer acts as an identifier and thus making the communication robust. Once PLC receives the oven number, it coordinates the other machines accordingly.

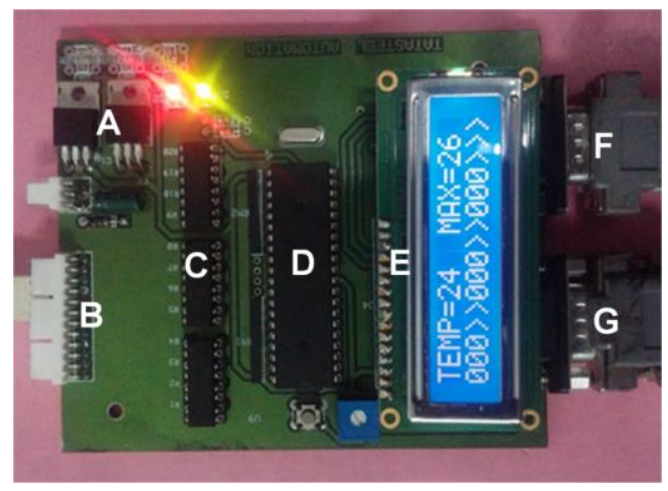

Fig. 5. Autospot communication card

Tab. 2. Different sections of the communication card

\begin{tabular}{|c|c|}
\hline Marker & Module \\
\hline A & Voltage regulator \\
\hline B & Input from IR sensors \\
\hline C & Opto-couplers \\
\hline D & MCU unit \\
\hline E & LCD Display \\
\hline F & RS232 / ISP \\
\hline G & RS485 to PLC \\
\hline
\end{tabular}

This device is implemented using in-house developed embedded platform built around ATMEL Microcontrollers. The processing speed of this embedded platform is 20MIPS (Million Instructions per second) with added features like on-board temperature sensor, LCD display and opto-isolated inputs as shown in Tab. 2.

Thus AUTOSPOT system enables coke plant machines, to be driven accurately to the defined positions. The system identifies the predefined position such as oven number, its alignment status with respect to the defined position etc.

\subsection{RF Based Oven identification System}

RFID tags (Fig. 6) are positioned on a wall alongside the locomotive track at the same distance as between coke oven door centres [1]. Machine is fitted with a sealed RFID antenna/Reader which is directional. This locates each locomotive to within $30 \mathrm{~cm}$ and the location is presented in the cab on a monitor. This location data is transmitted in real time to the central coke plant control room so that the precise location of each locomotive is shown.

This system consists of two parts. First part is the RF tags. These are encapsulated in high temperature casings (Flat rectangular plate structures). These structures are mounted in front of individual ovens. The tags are highly durable and withstand oil, water, grease, ice etc. They are powered by the reader via inductive coupling. The 1024 bit EEPROM memory of tags and cards is fast and easily read and programmed. The content of the memory can be permanently locked by the user in 16 bit blocks. Built-in functions such as 16 and 32 bit password, different read/write procedures, permanent locking of memory, 32 bit read only ID number etc. together with error-free data transfer ensure a high level of security.

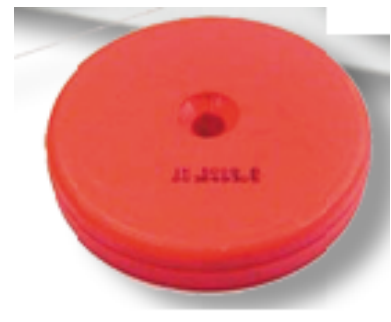

Fig. 6. RF Tags

In the second part, these readers are designed for RFID applications requiring good performance and reliability. The housing is suitable for outdoor usage and installed directly onto metal surfaces without additional insulation. An easy-to-use ASCII protocol is available for 'read' and 'write' commands. High speed, full duplex communication with RF tags is well secured. The data transmitted to the tag is echoed back to the reader for verification. The reader is enabled with automatic double read facility for additional verification of data.

\subsection{Imaging based Oven Identification}

This is a method for automatic identification of coke ovens for auto positioning systems in coke plants of steel industries. Image Processing techniques are more advantageous to that of using mechanical identification systems of coke ovens. The major limitation of using the mechanical arrangement is that, this system is placed at the bottom of the coke oven machines which is prone 
to damage due to heat, dust and even collision and other mechanical damages.

For the purpose of automatic identification of the coke oven no's, a specially designed MS/SS plate is placed on the coke ovens which contain rectangular slots. These slots represent the binary 1 's and 0 's and are used to identify the coke ovens, so that each coke oven has a unique identification number. This process of containing binary digits on a plate is known as binary encoding. Since these are solid mild steel /SS plates; there would be no major hindrance in the identification process due to harsh environment conditions (high temperature, dust and dirt accumulation). The identification process of the binary encoded plates is done by using the fundamental idea that the rectangular plate has a definite geometry and this geometry can be detected using edge detection algorithms. Following are the key ideas used in localisation of the binary encoded plates:

\subsubsection{Canny edge point detections}

The Process of Canny edge detection algorithm [2] can be broken down into 5 different steps:

1) Application of Gaussian filter to smooth the image in order to remove the noise. Since all edge detection results are easily affected by image noise, it is essential to filter out the noise to prevent false detection caused by noise. Gaussian filter To smooth the image, a Gaussian filter is applied to convolve with the image. This will slightly smoothen the image to reduce the effects of obvious noise on the edge detector. The equation for a Gaussian filter kernel of size $(2 \mathrm{k}+1) \times(2 \mathrm{k}+1)$ is given by

$$
\begin{array}{r}
H_{i j}=\frac{1}{2 \pi \sigma^{2}} \exp \left(\frac{(i-k-1)^{2}+(j-k-1)^{2}}{2 \sigma^{2}}\right) \\
\text { where, } 1 \leq i, j \leq(2 k+1)
\end{array}
$$

2) Finding the intensity gradients of the image

An edge in an image may point in a variety of directions. Four filters are used to detect horizontal, vertical and diagonal edges in the blurred image. The basic function of edge detection algorithms to determine horizontal and vertical intensity gradients, viz., $G_{x}$ and $G_{y}$. From this the edge gradient and direction can be determined as

$$
\begin{gathered}
G=\sqrt{G_{x}^{2}+G_{y}^{2}} \\
\text { and, } \theta=\operatorname{atan2}\left(G_{y}, G_{x}\right)
\end{gathered}
$$

3) Application of non-maximum suppression to get rid of spurious response to edge detection. NonMaximum suppression is applied to "thin" the edge. It is often an intermediate step in edge detection and refers to technique where direction of the edge is determined after comparing the edge strength of the neighbouring pixels in positive and negative direction of actual edge direction. This is preserved and further iterations build the edge trace.
4) Dual thresholds: Non maximum edge compression results in edge map that to some extent represent the true edges in the images. However, owing to noise and other variations in the images, it is likely that some actually 'non-edge' pixels being classified as edge pixels. Such pixels have strong gradient values. The following technique is used to remove these spurious edge pixels: If the edge pixel's gradient value is greater than the high threshold value, they are noted as strong edge pixels. Any edge pixel whose gradient value is smaller than the high threshold value and larger than the low threshold value is marked as weak edge pixels. A pixel will be rejected if the pixel value is smaller than the low threshold value. Empirically determined, two threshold values will be needed for identifying different images. True edge map may be obtained by suppressing all the weak edges in the image which are not directly connected with any of the strong edges.

5) Hough Transform: Low level image processing is helpful in determining edge points and thus contours of various shapes in the image. Hough transform [3] is one such mechanism that is based on grouping of edge pixels representing a possible line in the image space.

Line detection is the simplest case of Hough transform. Among many possible parametrized form of line, the Hesse normal from is preferred as it remains in bound for all possible inclinations and distances.

$$
r=x \cos \theta+y \sin \theta
$$

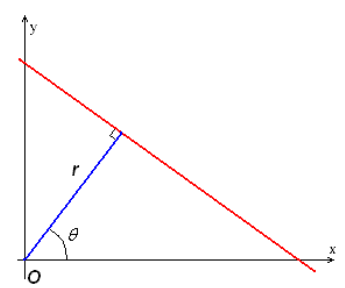

Fig. 7. Hough transform space

Where $r$ is the perpendicular distance from the origin to the straight line, and $\theta$ is the angle between the $\mathrm{x}$ axis and the perpendicular.

The linear Hough transform algorithm (Fig. 7) uses a two-dimensional array, called an accumulator, to detect the existence of a line described by $\mathrm{r}=x \cos \theta+y \sin \theta$. The accumulator size equals the number of unknown parameters, i.e. two, considering quantised values of $r$ and $\theta$ in the pair $(r, \theta)$. For each pixel at $(x, y)$ and its neighbourhood, the Hough transform algorithm determines the existence of enough evidence of a straight line at that pixel. If so it will estimate the parameters $(r, \theta)$ of that line and then go for the accumulator's bin that the parameters fall into, and increment the value of that bin.

The output of the Hough transform is a 2-D array consisting of accumulated values at various $(r, \theta)$ values. This can be interpreted as 2-D surface where the peaks in the surface truthfully represent 
the presence of a line in the image. As shown in an example below:
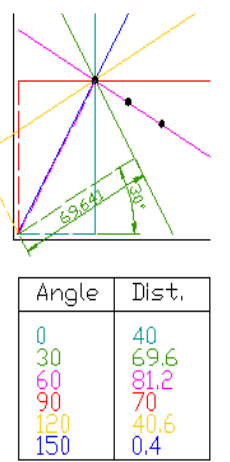
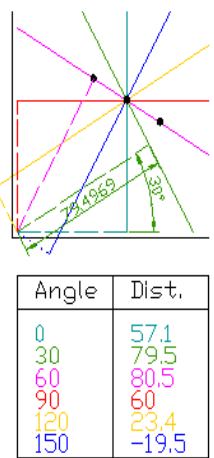
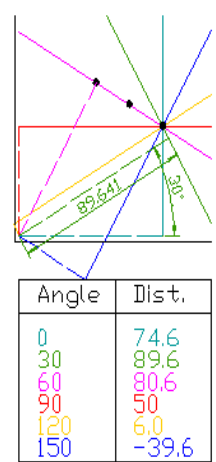

Fig. 8. Illustration of accumulator filling process

The output of the Hough transform is a 2-D array consisting of accumulated values at various $(r, \theta)$ values. This can be interpreted as $2-D$ surface where the peaks in the surface truthfully represent the presence of a line in the image. As shown in an example below:

Once all the lines in the images are determined, typically of the order of $10 \mathrm{~K}$, all possible 4combinations $\left(\left[\wedge^{\wedge} n\right] c_{-} 4\right)$ are made i.e., the number of quadrilaterals that can be made from $\mathrm{n}$ lines. Each combination passes through a scoring mechanism to determine its fitness as one truly representing the boundaries of the binary encoded plate. (Fig. 9) The line group with highest score is used to segment the image out of the cluttered scene background and the pattern extraction module precisely determines the position of 1 's and 0 's, which is then converted into decimal numbers.

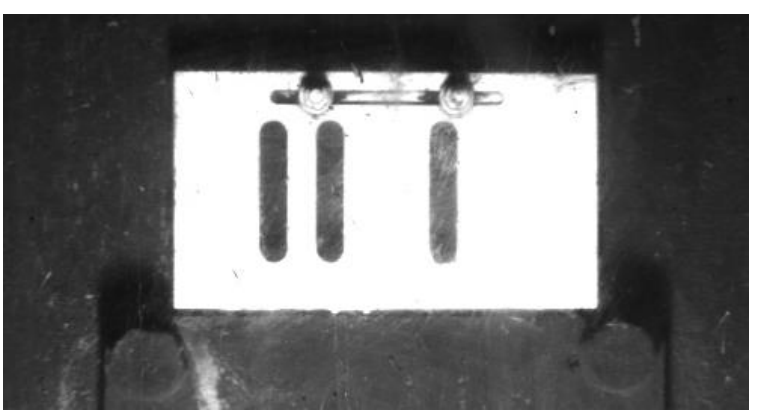

Fig. 9. Binary encoded SS plate

A camera with an enclosure is positioned inside the coke oven machine which can recognise the plate. For this, a template image of the slots is used and compared with the image used for such identification [4]. The camera has a frame rate of 120 frames per second and by this, the plate can be identified even when the door extraction car is moving. Hence, it is very advantageous to use such an application for coke oven identification. Main objectives are to find binary coded plates present in the field of view and to calculate the decimal equivalent of the binary coding. This application is based on the correlation search methodology in which template images are searched in base image at a particular scale. If right templates are found, then calculations are done with the Indexes to find out that whether the template images are found at the particular position. If presence of the plate is confirmed, then the equivalent decimal value is computed. Thus the coke oven no's can be identified by the unique numbers by which the slotted metallic plates can be identified. Furthermore, since this camera arrangement is made in the moving coke oven cars at coke plants, the complete cycle of coke ovens for logistical purposes can be determined and used for analysis.

\subsection{RADAR based oven identification system}

Advances in technology and new demands in growing industries require next generation of positioning techniques using wireless methods [5]. An absolute positioning system provides lot of opportunities in industry in terms of safety and man-less operations. LASER systems are precise but they suffer enormous inaccuracies in dusty environment and erroneous alignment. Conventional RADAR systems are rugged but prone to false echoes from various surrounding metallic structures usually present at industrial site [6] [7] [8]. This affects the measurement statistics greatly and limits the range to a few meters (50 meters) [9] [10]. Hence, an active RADAR based system has been designed and developed for its suitability in industrial conditions and is used in positioning requirements of rail borne vehicles.

The system operates in ISM band and measures distance using Return Time of Flight (RTOF) method. The echo signal is a coded active return generated by the slave to measure distance with very high precision and accuracy [11]. Using this method the distance between the stations can be accurately determined over a range of 500 meters. The block diagram of the developed RADAR system is shown in Fig. 10.

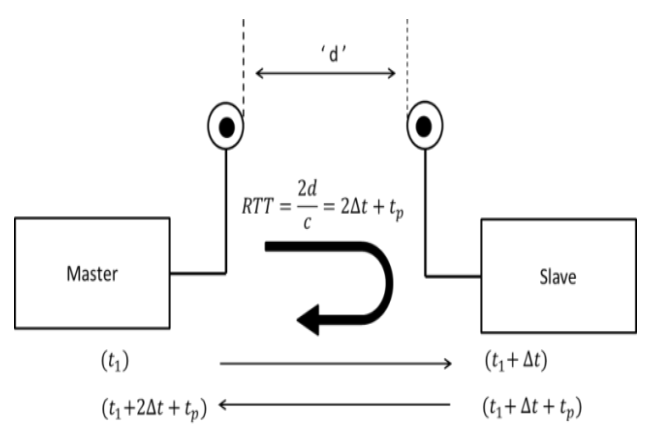

Fig. 10. Block diagram of the developed RADAR system with Master (base-station) and Slave (transponder). Round Trip Time (RTT) is calculated as shown, from which distance can be measured accurately 


\subsubsection{Design cycle}

System design cycle started at a proof of concept level, where the circuits were designed on test boards in laboratory setup Fig.11. Both the units were developed and power levels were tested. Modifications required on theory based design were implemented and the proof of concept was established.

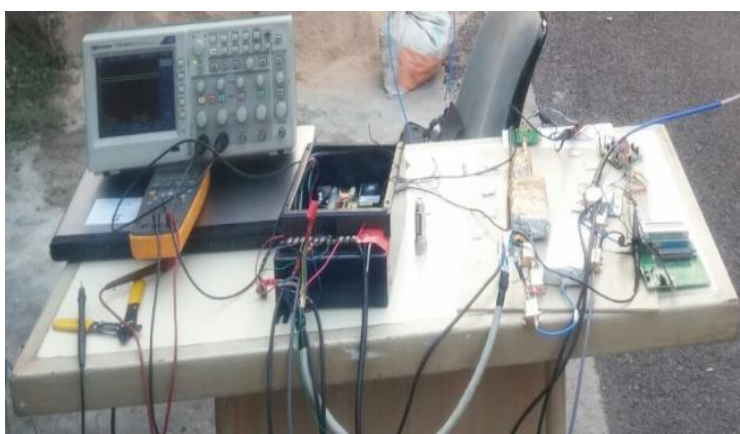

Fig. 11. Proof of Concept established. Circuits designed on test boards and connector based modules

This system was designed and implemented keeping practical constraints in mind. Different modules were grouped together and segregated into layers (Fig. 12). Shielding techniques and filters were implemented to reduce the noise level of the system. Based on the observations and associated inferences redesign/modifications of system was done to improve the accuracy and range. As a step towards creating the product the system was implemented at a prototype level [12].
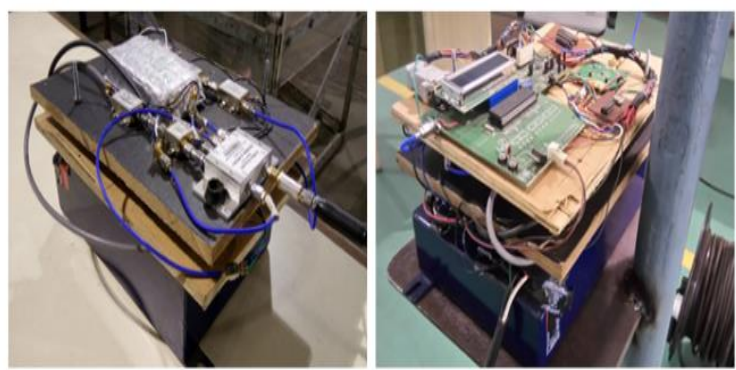

Fig. 12. Multi-layered Base station and

Transponder. Considerable reduction in size and product can be seen shaping form

The design started with a printed circuit board (PCB) having small form factor, as this would directly impact the system dimensions. To implement the positioning system in plant environment it has been made mechanically stable and robust [13, 14, 15]. For this purpose all the loose wires and signal connected cables have been removed completely. A single layer double sided PCB was designed and simulated with additional features like AGC, temperature compensation circuitry and RS485 communication link built into it. Fig. 13 shows the actual photograph of the inhouse developed system. The radar module consists of three major blocks e.g. RF module, Signal processing unit and power supply (Tab. 3). The whole assembly has been encased in a machined aluminium block (Fig. 14).

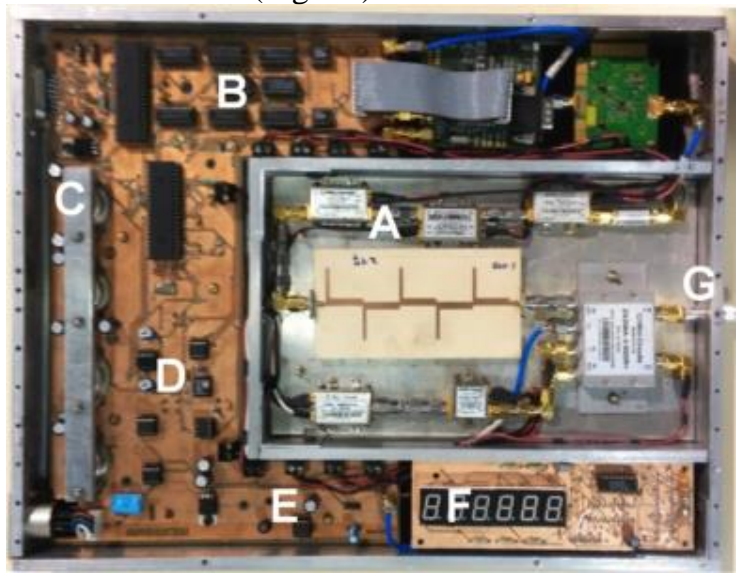

Fig. 13. Final product layout (Base Station). A single PCB was designed along with the RF components with proper shielding. Final output display is also indicated

Tab 3. Different modules of Final Product developed

\begin{tabular}{|c|l|}
\hline Marker & \multicolumn{2}{|c|}{ Module } \\
\hline A & RF transceiver \\
\hline B & Signal processing unit \\
\hline C & Power supply \\
\hline D & AGC unit \\
\hline E & RS485 output \\
\hline F & Display \\
\hline G & To antenna \\
\hline
\end{tabular}
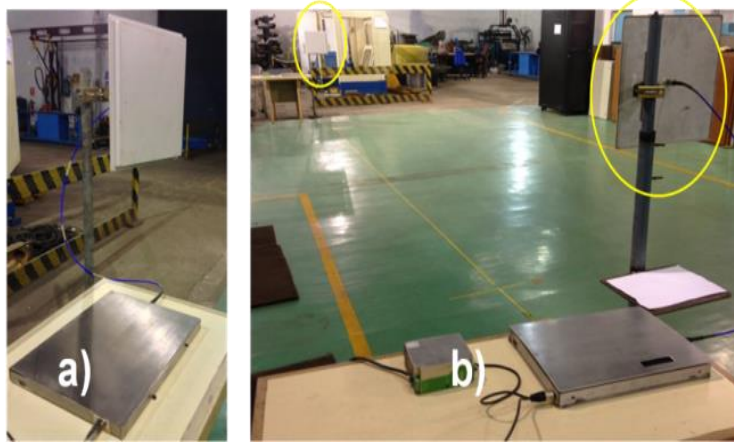

Fig. 14. Actual Photograph of the Radar System a) transponder station with antenna b) base-station with antenna facing the transponder

\subsubsection{Field Test}

To validate this system in the plant environment a field trial was conducted by installing the system on moving machines at Coke ovens. The trial setup (Fig. 15) consists of the RADAR system (test equipment) with two active units, one kept at fixed location (quenching tower) and the second one on the CTC machine. Antennas were mounted on either end ensuring line of sight between them. A LASER Distance Meter (used as reference unit) has also been installed parallel to this system for initial calibration and subsequent validation of the system (Fig. 16). The distance reading of the CTC from quenching tower has been measured for both these instruments during LT (Long Travel). 


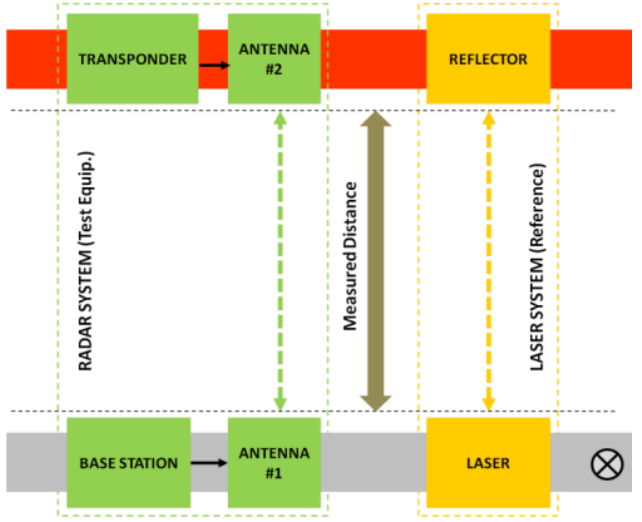

Fig. 15. Block Diagram of trial setup: Radar system (coloured Green) consists of the Base station in fixed location and Transponder in moving platform. Measurement result (distance) is obtained at Base station (quenching tower). Laser distance meter is also placed alongside, with its reflector at moving platform side, giving out reference reading for validation

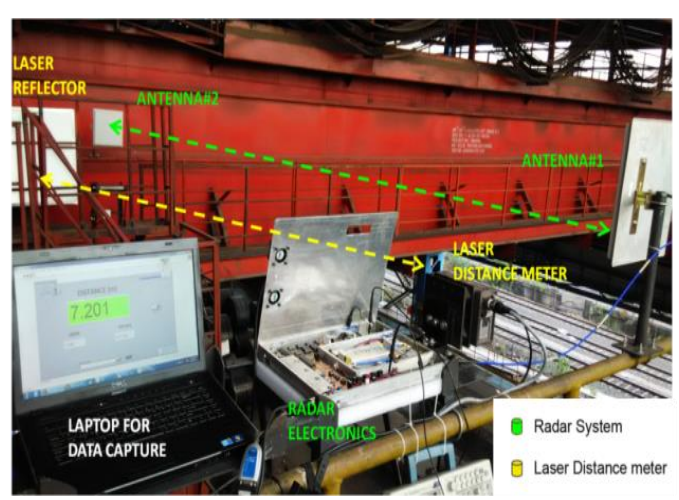

Fig. 16. Actual photograph of the installation, Radar (along with antennas) and Laser systems mounted in parallel. A laptop communicates with both systems and is used for data recording

\subsubsection{System Calibration}

Radar based positioning system developed in house measures distance by measuring the return time of flight (RTOF method). The output data obtained was time value which would be converted to distance reading. This required a onetime calibration, which was performed with the help of Laser Distance Meter. Upon plotting a graph between the measured time values and reference distance values (from LDM), the coefficients were obtained for converting time to distance (Fig. 17). This also removed all offset errors with respect to reference.

Linearity of this plot was essential as it is the primary requirement for any measuring instrument. As can be seen, the plot obtained shows excellent linearity (with negligible second order term). Also during each of the readings, it was observed that the measurements through multiple random processes followed Gaussian distribution.

\subsubsection{Validation}

Once the calibration coefficients were obtained and fed into the system, absolute distance readings were obtained from Radar. Validation of these values was done by comparing them with the reference distance readings from the Laser System. Simultaneous readings of Radar and Laser were stored into a file. A plot has been generated for multiple measurements carried out during the movement of machine. The distance of the machine from quenching tower has been measured by both these instruments and plotted side by side. This plot shows excellent agreement between the measured distance readings from the RADAR and LASER system (Fig. 18).

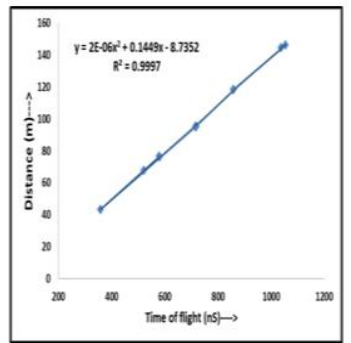

Linear plot between Reference distance (Laser) \& measured time of flight (Rodor). This plot provides the processes necessary coefficients for callibration.

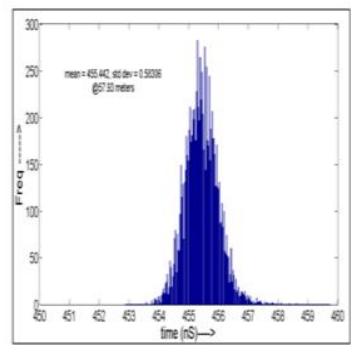

Distribution of row time of flight doto obtained. Goussion distribution is a result of all random in measurement link.
Fig. 17. Observations during System Calibration

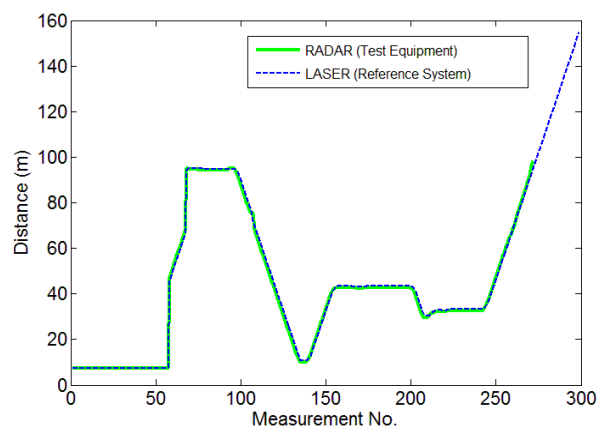

Fig. 18. Figure shows distance measured using Radar (Green) and Laser (Blue), during normal movement of moving machine w.r.t. quenching tower. About 300 readings were taken

(Measurement \#) and distance was measured from both systems. It was observed that the recorded distances shows high degree of correlation during the entire period

\section{COMPARATIVE STUDY}

a) Opto mechanical system (Marker plate reader/Sensor [AUTOSPOT] based Oven Identification): In this system slotted plates which are binary coded, are placed in front of each oven. The reader units are placed on the machine.

The advantages of this system is that it is very accurate and almost one hundred percent accurate. It is proven to be rugged and cost effective too.

As regards its disadvantages the prerequisite for this system to operate efficiently is the track linearity and levelling. The smooth movement of 
the machines on the track is also essential for this system to perform up to the mark. Perfect alignment is mandatory.

b) RF Based Oven identification System: In this technique, RF tags are placed in front of each oven. $\mathrm{RF}$ reader is placed on the machine.

The advantages of this system are that its alignment is not critical. It can be also read from a distance.

The disadvantage of this system is the following. Interference from nearby tags can occur if placed very closely. High temperature tags are not easily available. These tags cannot withstand mechanical collision. Deposition of conductive coal dust on them forces the system to malfunction.

c) Imaging based Oven Identification: For this system marked slotted plates are installed in front of each oven and the camera is placed on the machine.

The advantages of this system are that it is cost effective and nearly maintenance free.

The disadvantages of the system are the following. Presence of PC makes it vulnerable, considering the PC needs to be kept in the moving machine. The glass in front of the camera should be kept very clean providing clear path of sight. Tags should be regularly cleaned, painted. Requirement of cleanliness of tags and continuous power supply to protect the PC against fluctuations should be guaranteed.

d) RADAR based oven identification system: Here in this system two RADAR transceivers are mounted on two different machines face to face. Distance between the machines is measured on a continuous basis.

The advantage of this system is continuous measurement of distances .The system is also maintenance free one.

As regards disadvantages the system is costly. It is not very accurate in determining exact position. It has heavy dependence on the geometry of the Coke Oven.

\section{CONCLUSION}

This paper presents different automatic identification methods of coke ovens for auto positioning systems and machine interlocking systems in coke plant of steel industries. Initially the paper describes the different established methods for automatic identification of coke ovens. Reviewing the existing methods the problem has been identified. A Radar based oven identification system has been proposed to address the problem. The implementation steps of the proposed system have been elaborated with the customised developed setup. The analysis of the implemented system has been shown with a comparative statement for assessing the potential of the proposed system. The results and comparative analysis indicate the promising prospective of the system over the existing methods.

\section{REFERENCES}

1. Ting SL, Kwok SK, Albert H, Tsang C, George T, Ho $\mathrm{S}$. The study on using passive RFID Tags for indoor positioning. International Journal of Engineering Business Management. 2011; 3(1): 9-15. https://doi.org/10.5772/45678

2. Canny J. A computational approach to edge detection. IEEE Trans. Pattern Anal. Mach. Intell. 1986;8(6).

3. Xu L, Oja E, Kultanen P. A new curve detection method: Randomized hough transform (RHT). Pattern Recognition Letters, 1990; 11(5): 331-338.

4. Patent application no 705/KOL/2011, A Method for Automatic Identification of Coke Ovens for Auto Positioning Systems in Coke Plant of Steel-Industries

5. Urruela A, Sala J, Riba J. Average performance analysis of circular and hyperbolic geolocation. IEEE Trans. Veh. Technol. 2006; 55(1): 52-66. https://doi.org/10.1109/TVT.2005.861172

6. Vossiek M, Wiebking L, Gulden P, Wieghardt J, Hoffmann C, Heide P. Wireless local positioning. IEEE Micro. 2003; 4(4): 77-86.

7. Liu H, Darabi H, Benrjee P, Liu J. Survey of wireless indoor positioning techniques and systems. IEEE Trans. Syst., Man, Cybern. C, Appl. Rev. 2007; 37(6): 1067-1080. https://doi.org/10.1109/TSMCC.2007.905750

8. Zhang C, Kuhn M, Merkl B, Fathy AE, Mahfouz M. Accurate UWB indoor localization system utilizing time difference of arrival approach. in IEEE Radio Wireless Symp., San Diego. 2006: 515-518. https://doi.org/10.1109/RWS.2006.1615207

9. Stelzer A, Pourvoyeur K, Fischer A. Concept and application of LPM - A novel 3-D local position measurement system. IEEE Trans.Microw. Theory Tech. 2004; 52(12): 2664-2669.

10. Kossel M, Benedickter HR, Peter R, Bachtold W. Microwavebackscatter modulation systems, in IEEE MTT-S Int. Microw. SympDig. Boston. 2000:14271430.

11) Vossiek M, Gulden P. Switched injection locked oscillator: Anovel versatile concept for wireless transponder and localization systems. IEEE Trans. Microw. Theory Tech. 2008; 56(4):859-866. https://doi.org/10.1109/TMTT.2008.918158

12. Patent application no 201631010714, Radar Based Positioning System.

13. Vossiek M, Urban A, Max S, Gulden P. Inverse synthetic apertures econdary radar concept for precise wireless positioning. IEEETrans. Microw. Theory Tech. 2007; 55(11): 2447-2453. https://doi.org/10.1109/TMTT.2007.908668

14. Roehr S, Gulden P, Vossiek M. Method for high precision clock synchronization in wireless systems with application to radio navigation. Radio and Wireless Symposium, 2007: 551-554. https://doi.org/10.1109/RWS.2007.351890

15. Roehr S, Vossiek M, Gulden P. Method for high precision radar distance measurement and synchronization of wireless unit. Microwave Symposium. IEEE/MTT-S Intern., IEEE. 2007: 13151318. https://doi.org/10.1109/MWSYM.2007.380436

Received 2018-01-25

Accepted 2018-04-23

Available online 2018-04-26 


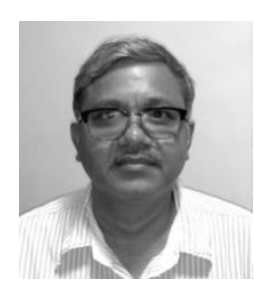

Prabal PATRA received the B.Tech. degree in Instrumentation and Electronics engineering from the Jadavpur University, Kolkata, in 1992. He is currently pursuing the Ph.D. degree with Jadavpur University. He is currently Head of the department of Instrumentation \& Control at Automation Division of Tata Steel, which is a R\&D department. His research areas include Control systems, Machine Vision, Embedded systems, RF \& Microwave Technology.

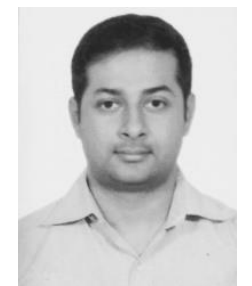

Chitresh KUNDU received his M.Tech. degree from IIT Kharagpur, India, in 2010. He is currently working as Senior Technologist (Instrumentation and Control, Automation Division, TATA Steel. $\mathrm{He}$ is responsible for development of various embedded system and RADAR based application for TATA Steel. His research interests include the fields of antennas, RADARS, embedded systems, IOT platforms.

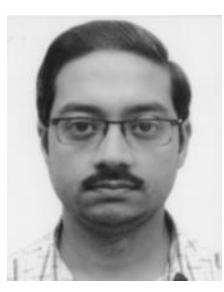

Arunangshu GHOSH received the Ph.D. degree from Jadavpur University, Kolkata, India, in 2015. $\mathrm{He}$ is currently an Assistant Professor with the Department of Electrical Engineering, National Institute of Technology, Patna, India. His research interests include electronic tongue, machine olfaction, quartz crystal microbalance sensors, and pattern recognition.

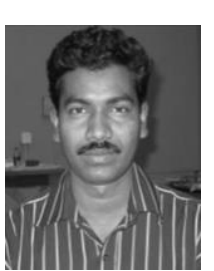

Bipan TUDU received the Ph.D. degree from Jadavpur University, Kolkata, India, in 2011. He is currently a Professor with the Department of Instrumentation and Electronics Engineering, Jadavpur University. His research interests include pattern recognition, artificial intelligence, machine olfaction, and the electronic tongue.

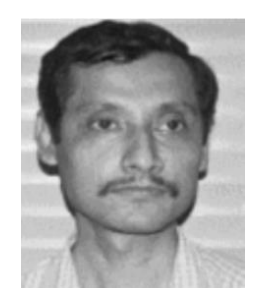

\section{Rajib BANDYOPADHYAY}

received the Ph.D. degree from Jadavpur University, Kolkata, India, in 2001. He is currently a Professor with the Department of Instrumentation and Electronics Engineering, Jadavpur University, and a Research Professor with the Laboratory of Artificial Sensory Systems, ITMO University, Saint Petersburg, Russia. His research interests include the fields of machine olfaction, electronic tongue, and intelligent systems. 